\section{ACERCA DE UNA GENTRIFICACIÓN "PLANETARIA", POLÍTICAMENTE ÚTIL*}

Ernesto López Morales**

\section{Resumen}

En todo el mundo los centros urbanos se "redescubren" para objetivos de reproducción de capital, una recomposición que conlleva la inyección de altos niveles de inversión privada para el desarrollo de nuevos espacios, servicios y vivienda orientados a segmentos medios y altos, lo que pareciera ser consustancial con la fase financiarizada del capitalismo urbano. En este contexto, la teoría de la gentrificación se debate actualmente entre ser una explicación útil para desarrollar un campo de investigación comparativa, en base a definiciones genéricas del proceso, o bien adoptar un enfoque de raíz post-colonial "provincializado" que rechaza la gentrificación por ser supuestamente una narrativa estructural proveniente del norte global inaplicable en el sur global. En este ensayo, argumento la necesidad de avanzar en una episteme que separe los aspectos contingentes y en cambio desarrolle

\section{ABOUT A POLITICALLY USEFUL "PLANETARY" GENTRIFICATION*}

Ernesto López Morales**

\section{Abstract}

There is a global trend towards the "rediscovery" of urban centers for capital reproduction purposes. Such an exercise involves high levels of private investment for the development of new spaces, services and dwellings aimed at middle and higher-income segments, thus suggesting a close relationship with the financial phase of urban capitalism. In this context, gentrification theory is currently regarded either as an explanation that allows the development of the field of comparative research, based on generic definitions about the process, or through adopting a post-colonial 'provincial' approach that rejects the very concept of gentrification as a structural narrative that comes from the Global North inapplicable to the Global South. This paper suggests the need to develop an episteme intended 
en mayor profundidad los cruciales aspectos espaciales, políticos, económicos y sociales referidos al fenómeno de la gentrificación. Ejemplifico en base a un análisis de las ciudades de Santiago y Ciudad de México, además de proveer revisión teórica actualizada, referida a otras ciudades mundiales. Concluyo que los procesos de gentrificación parecieran ser convergentes y genéricos a nivel planetario, en un contexto de mayor movilidad y agresividad del poder político-financiero.

\section{PALABRAS CLAVES: GENTRIFICACIÓN; ENFOQUE POSCOLONIAL; BRECHA DE RENTA; BLANQUEAMIENTO; CIUDAD DE MÉXICO; SANTIAGO DE CHILE}

Recibido: 08-03-2016

Aceptado: 30-09-2016

* $\quad$ Este trabajo fue apoyado por los proyectos Fondecyt 11100337 Contested Cities Research Network Scheme FP7-PEOPLEPIRSES-GA-2012-318944 y CONICYT/FONDAP COES 15130009.

* Chile. Académico del Departamento de Urbanismo de la Facultad de Arquitectura y Urbanismo, Universidad de Chile. Correo electrónico: elopez@uchilefau.cl. to separate factual aspects and explore some key spatial, political, economic and social elements associated with the gentrification phenomenon. Such an exercise is based on the analysis of two cities, Santiago and Mexico City, as well as from an updated theoretical review, referring to other world cities. It is concluded that gentrification processes at planetary level seem to converge and share the same characteristics within a context marked by the greater mobility and aggressiveness of political-financial power.

KEYWORDS: GENTRIFICATION; POST-COLONIAL APPROACH; RENT GAP; WHITENING; MEXICO CITY; SANTIAGO, CHILE

Received: 08-03-2016

Accepted: 30-09-2016

* This paper is based on the Fondecyt project 11100337; Contested Cities Research Network Scheme FP7-PEOPLEPIRSESGA-2012-318944 and CONICYT/FONDAP COES 1513009.

* Chile. Academic, Department of Urban Planning, Faculty of Architecture and Urban Planning, University of Chile. Email: elopez@uchilefau.cl. 


\section{Introducción}

En la última década, el enfoque "postcolonial" en investigación social urbana ha ganado relevancia. Este enfoque ofrece interpretar de manera contextualmente apropiada distintos regímenes urbanos en el mundo. Autores "postcoloniales" como Roy, McFarlane y Robinson ${ }^{1}$ han construido un verdadero campo epistemológico "provincializado", es decir, escéptico de la transferencia teórica desde el norte al sur global (o del oeste al este, como se quiera), bajo el argumento de que ello no sólo desconoce sino también impide el desarrollo de teorías nuevas, variopintas, contextualmente situadas, más honestas y apropiadas al entorno desde donde se extraen, en diferentes lugares, momentos y realidades. Una postura postcolonial debiese contribuir al florecimiento de una teorización más ágil y flexible respecto a la de décadas pasadas.

Este llamado postcolonial exige también esfuerzos comparativos mayores. El enfoque es escéptico frente a definiciones teóricas totalizantes (podríamos decir isotópicas) y por lo general critica prácticamente todas las teorías existentes explicativas del funcionamiento de la economía política del capitalismo. Los autores mencionados invitan a socavar un sistema existente de construcción

1 Roy, 2009; McFarlane, 2010; Robinson, 2011; McFarlane y Robinson, 2012. hegemónica, dominado por los aparatos productores de conocimientos del norte global (aunque raramente se pronuncian acerca de las élites intelectuales del sur global, gobiernos, universidades, think tanks, cuerpos académicos, etc., como si no fueran muchas veces fuerzas regresivas presentes en los contextos locales). En rigor, se trata de un enfoque tributario del post-estructuralismo más radical, aggiornado para el siglo XXI en su sentido más interpretativo, que circunscribe la teoría dentro de un cierto territorio heterotópico y delimitado geográficamente. Siguiendo a la filóloga Spivak ${ }^{2}$, autores como Roy, Robinson y McFarlane abogan por un "des-aprendizaje", una provincialización del conocimiento urbano, para en cambio dejar florecer las explicaciones emanadas del campo, la fenomenología de lo local, un gran sí a un variopinto argot regional para denominar procesos, etc. El cuestionar la validez interpretativa de la teoría de la gentrificación ha llegado a ser un buen ejemplo de este debate postcolonial anti "difusionista", ya que el enfoque postcolonial entiende la gentrificación como una imposición teórica que se difunde desde el norte al sur global.

Utilizando este tipo de lógicas, algunos autores infieren varios problemas epistemológicos al tratar el concepto de gentrificación a escala "planetaria"3.

\footnotetext{
2 Spivak, 2003.

3 Ver Maloutas, 2011; Betancur, 2014 o Ghertner, 2015 y su estudio sobre Bombay en la India.
} 
Son escépticos frente al aporte del concepto para explicar el cambio urbano en distintas ciudades del mundo. Con matices, estos autores sostienen la inaplicabilidad de las variables que supuestamente caracterizan el fenómeno en lugares que no sean del hemisferio norte. Maloutas sostiene que para hablar de gentrificación debe existir, en todos los casos y como variable causal principal, un cierto tipo definido de agente de alto capital económico y cultural, una sujeto gentry clase media generador de un patrón de consumo particular y poseedor de una imagen predeterminada reconocible. Ghertner y Maloutas sostienen asimismo que la gentrificación se circunscribe a un momento particular post-industrial, en el norte global, idea también ya expresada previamente por Hamnett ${ }^{4}$, que explicaba la gentrificación de las ciudades inglesas como correlato del ascenso de una nueva clase media post-industrial con mayor poder de consumo y con escasa conciencia de clase, algo circunscrito a determinado momentos de desarrollo histórico y geográfico.

Ghertner ${ }^{5}$ plantea que, al hablar de recambio urbano, hay que mirar más allá de las fuerzas puramente económicas, y que la gentrificación debería ser un relato en sí mucho más rico que la simple discusión en torno a los conflictos sobre las "curvas de valor de la tierra". Estando plenamente de acuerdo con este autor en esa premisa, estimo errónea su conclusión: Ghertner invita a "desaprender", es decir, desvirtuar lo que ya sabemos acerca del funcionamiento y reproducción del capital en la ciudad, y lo que ha sido explicado acerca de la expansión del capitalismo urbano, sus ciclos de crecimiento y crisis, y sus muchas injusticias derivadas de la especulación inmobiliaria en barrios centrales. Me parece evidente la necesidad de comprender las estructuras capitalistas que generan gentrificación a la par de las particularidades sociológicas, culturales e institucionales que el fenómeno adquiere en cada lugar, sin por ello perder la noción más política y socio-económica del concepto.

El problema de Ghertner, Maolutas y los autores postcoloniales citados, es que parecieran basarse en una definición superficial histórica de la gentrificación. Es decir, una cosa es criticar la trayectoria difusionista, pero otra muy distinta es el rechazar todo el concepto de gentrificación a nivel global, simplemente porque los casos de polarización de clase asociados al redesarrollo capitalista en distintos barrios del mundo no se "parecen" a las descripciones tradicionales de gentrificación vistas por primera vez en el norte global en la década de 1960 por Ruth Glass. Es decir, desconocen el fenómeno y se quedan sólo con las apariencias del fenómeno, asumiéndolas per se como definiciones

4 Hamnett, 1991.

5 Ghertner, 2015. 
totalizantes. Este es un error en el que muchos estudiosos caen, un riesgo de caer en un conjunto clichés formales y reflexiones dispersas, en lugar de una vibrante comunidad de pensamiento y de un proceso de aprendizaje comparado.

El problema que veo en la crítica postcolonial actual a la teoría de la gentrificación es que no entiende que la gentrificación se trata, por lo general, de un proceso que guarda una relación muy estrecha con el funcionamiento del capitalismo, y como tal puede ser explicada. La gentrificación asume también diversas manifestaciones sociales y físicas dependiendo del contexto, pero siempre con el efecto de producir desplazamiento o exclusión desde el centro urbano de los segmentos sociales más bajos. Esto quiere decir que el riesgo de los enfoques postcoloniales radicales es que eliminan innecesariamente una perspectiva rica en crítica urbana que por décadas ha aglutinado una masa voluminosa (siempre insuficiente) de académicos caracterizados por una perspectiva económicopolítica, y que han tratado de articular, a través de explicaciones teóricas, las injusticias de la elitización capitalista de la ciudad. Esta es la primera premisa del presente artículo.

La segunda premisa del artículo es que la gentrificación es un concepto políticamente maleable y utilizable por el activismo urbano, e útil para confrontar las numerosas injusticias devenidas del desarrollo urbano neoliberal. La teoría de la gentrificación ofrece también una articulación empírico-teórica con otros campos de teorización altamente vigentes, como son el derecho a la ciudad, los movimientos sociales, el funcionamiento del Estado neoliberal, las elites, la incidencia de las políticas de transporte en la valorización comercial del suelo, la exclusión social, entre las más importantes. Ello, sin perder identidad y aplicabilidad el concepto necesariamente.

\section{Metodología y contexto de casos}

Las dos secciones posteriores explican ambas premisas del artículo respectivamente. La primera sección se basa en una exhaustiva revisión teórica previa del autor, como parte de la edición reciente de diversos volúmenes vinculados al tema junto a un equipo internacional de investigadores ${ }^{6}$. Ello implicó también la organización de dos talleres de análisis y discusión de casos, uno desarrollado en la ciudad de Londres en marzo de 2012, y el segundo en Santiago, en abril de 2012.

La segunda sección son conclusiones emanadas de un trabajo empírico desarrollada en las ciudades

6 Lees, Shin y López Morales, 2015, 2016; Shin, Lees y López Morales , 2016; López Morales, Shin y Lees, 2016. 
de Santiago y Ciudad de México. Para Santiago se utilizaron variables de corte cuantitativo, obteniéndose datos de panel de medición de márgenes de renta capturada por inmobiliarias y residentes propietarios $^{7}$ entre los años 2000 y 2012, y una encuesta aplicada el año 2012 a 746 hogares residentes "antiguos" de seis barrios centrales metropolitanos de Santiago ${ }^{8}$ en proceso de activa explotación inmobiliaria, en las comunas de Santiago, Recoleta, Independencia, Quinta Normal, Macul y Nuñoa. Esto, sumado a numerosas entrevistas con actores locales participantes, tanto a nivel de vecinos como de autoridades municipales e inmobiliarias. Al tener el presente artículo un objetivo de alcance teórico, las referencias metodológicas más específicas se encuentran en los artículos y libros citados del autor.

Para Ciudad de México, se aplicó una metodología diferente, en virtud de la mayor dificultad de acceder a los datos y costos de trabajo de terreno para los investigadores. Se aplicó un estudio de

7 López Morales, 2016a, 2016b.

8 López Morales, Arriagada, Gasic y Meza, 2015. antecedentes bibliográficos, documentos públicos y registros de prensa, sumado a una metodología de observación participante en la colonia Benito Juárez ${ }^{9}$, en dos instancias de dos semanas cada una, en los meses de abril de 2015 y febrero de 2016, por parte del autor y colaboradores académicos locales. El levantamientos de la información consistió en notas de campo, realización de un grupo focal, y entrevistas semiestructuradas a diversos actores, tales como vecinos de la colonia Juárez y otras colonias centrales, actores púbicos e inmobiliarias. Se realizó también un taller en noviembre de 2015 en Santiago, donde se discutieron aspectos centrales del proceso de recambio espacial en la colonia Juárez, participando el equipo de investigadores chilenos y la contraparte académica mexicana.

9 La colonia Benito Juárez se localiza en la delegación central Cuauhtémoc, tiene su origen durante fines del siglo XIX y estuvo destinada a población de clase alta. Hacia la segunda década del siglo XX, el barrio fue gradualmente abandonado por la élite, lo que propició la llegada de población de bajos ingresos, mezclada con sectores de población de clase media, artistas e intelectuales. Las consecuencias del sismo de 1985 profundizaron el deterioro del barrio, aunque en la actualidad la colonia es reconocida en la Ciudad de México por su activa dinámica inmobiliaria, persistiendo diversos inmuebles con irregularidad en la tenencia e instancias de organización vecinal para promover el arraigo y la permanencia de sus habitantes populares. 


\section{La gentrificación se ha vuelto "planetaria"}

La pregunta acerca de la aplicabilidad del concepto de gentrificación fuera del hemisferio norte no es nueva. El término "gentrificación" ha sido adaptado conceptualmente desde hace más de dos décadas para adoptar una trayectoria similar en todo el mundo ${ }^{10}$. En la actualidad vemos por el creciente número de casos bien documentados, que la gentrificación se está convirtiendo fundamentalmente en un fenómeno planetario si se considera de forma genérica ${ }^{11}$. El término "planetario" (en lugar de "global", teóricamente moribundo ${ }^{12}$ ); es útil para analizar los conflictos de clase asociados a las transformaciones urbanas actuales que responden a categorías mundiales tales como circuitos transnacionales de capital financiero, migraciones sociales, flujos de información y recursos, posicionamiento de ciertas ciudades y sus atributos a nivel internacional, transferencia o réplicas de políticas públicas, etc., sin caer en la lógica "difusionista" norte-sur ni las "olas" o "expansión neoliberal" de la gentrificación.

La gentrificación ayuda a entender también el hecho de que la idea de la "fijación espacial" (spatial

10 Véase Jones y Varley, 1994; Hackworth y Smith, 2001; Smith, 2002.

11 Lees, Shin y López Morales 2015, 2016.

12 Lees, Shin y López Morales, 2016. fix) como un remedio para la crisis de la sobre-acumulación no es suficiente explicación para regiones como China ${ }^{13}$, donde el exceso de acumulación en el circuito primario de la producción industrial va de la mano con el aumento de la inversión en el circuito secundario del entorno construido, al menos hasta la reciente crisis financiera de ese país. Cada uno de estos procesos refuerzan el avance del otro; el monopolio estatal de los instrumentos financieros proporciona interesados del sector privado con el poder de obtener mayores ganancias. Y está claro que la reconstrucción capitalista de las ciudades en el sur toma diferentes formas, pero esto no significa que este proceso no sea gentrificación. En la mayoría de los casos, la gentrificación es la superimposición de los estratos dominantes de reglas societales y de mercado, en espacios donde otras estructuras sociales alternativas las han precedido por décadas e incluso siglos.

Por ejemplo, son componentes de procesos de gentrificación los programas de pacificación y turismo en favela de Río de Janeiro, que van acompañados de una política explícita de desplazamiento de clases subalternas en especial en las favelas de Vidigal y Babilonia ${ }^{14}$; el proceso de "turistificación" altamente mercantilizado de Cartagena de Indias"15;

\footnotetext{
Shin, 2014.

4 Gaffney, 2015; Cumming, 2015.

15 Posso, 2015.
} 
la declaratoria de barrios centrales estratégicos para políticas de reestructuración metropolitana y vigorización de mercados financieros en Ciudad de México $^{16}$, donde subsisten numerosas propiedades intestadas que son gradualmente reemplazadas por regímenes formales de propiedad, consustanciales a las nuevas demandas de clases más poderosas; la eliminación forzada de vendedores ambulantes del centro de las ciudades de México ${ }^{17}$ o Quito desde la década de 1990; la higienización del espacio con el fin de atraer a los turistas que conducen a "turistificación"18 entre otros ejemplos complejos y variados de apropiación simbólica y material de clase en el espacio. No sólo son ejemplos de gentrificación, sino también formas agresivas de recobrar control de clase sobre el espacio urbano recentralizado.

Lees, Shin y López-Morales ${ }^{19}$ han corroborado que los procesos de gentrificación en ciudades de todo el mundo producen pluralmente distintos espacios de acumulación de capital en territorios urbanos, suburbanos y rurales; formales e informales. Esto es evidente en Seúl ${ }^{20}$, Santiago ${ }^{21}$, Washington

\author{
Gaytán y Pimentel, en prensa. \\ Delgadillo, 2014. \\ Janoschka, Sequera y Salinas, 2014. \\ Lees, Shin y López Morales, 2016. \\ Shin y Kim, 2016.
}

López Morales, 2010, 2011, 2013, 2015, 2016 b.
$\mathrm{DC}^{22}$, Londres ${ }^{23}$ y Bombay ${ }^{24}$, por citar cinco ejemplos variopintos; en esas ciudades, la renovación excluyente de barrios de bajos ingresos está siendo uno de los problemas político-económicos y sociológicos más relevantes relacionados con la vivienda. Las desigualdades asociadas a la gentrificación no son algo acerca de lo que los estudiosos simplemente piensan; se trata de algo que los residentes y activistas locales sufren a diario y ya conocen. El papel del Estado en la producción de aumento de la segregación urbana ha sido hasta ahora insuficientemente conceptualizado por los académicos, pero vemos hoy en todas partes cómo los gobiernos de las ciudades del sur global se excitan ante la posibilidad de dirigir procesos de recambio urbano donde la inversión privada esté asegurada por márgenes altos de ganancia, es decir, procesos de reproducción y acumulación de capital, mientras que se rehacen los entornos construidos hasta poco antes desatendidos por la política pública.

La gentrificación es más que un simple despojo de valor del suelo, como incorrectamente argumenta Ghertner ${ }^{25}$. Se trata de la pérdida del valor de uso que la sociedad le ha otorgado al suelo urbano, es decir la conformación de un cierto paisaje urba-

$\begin{array}{ll}22 & \text { Mueller, } 2014 . \\ 23 & \text { Lees, 2014. } \\ 24 & \text { Doshi, 2013. } \\ 25 & \text { Ghertner, 2015 }\end{array}$


no, la riqueza simbólica y cultural del espacio, los recursos ambientales con que cuenta determinada localización (dotación de agua y cloacas, parques, hospitales, escuelas, por ejemplo), y el acceso a la movilidad y los servicios públicos utilizados y muchas veces en parte conseguidos o gestionados por los pobres que habitan en lugares urbanos que ahora se consideran deseables para objetivos políticos y económicos de rentabilización.

Ghertner planteaba en el 2015 que la gentrificación y acumulación por desposesión son dos cosas distintas. Está equivocado. La acumulación por desposesión o despojo es la creación de valor de cambio a partir de activos no comodificados socialmente utilizados por una sociedad determina$\mathrm{da}^{26}$. La gentrificación en muchos lugares del sur global es una forma de acumulación por desposesión, sobre todo cuando las empresas inmobiliarias, los planes de actuación urbanística, o ambos concertados, se focalizan en la transformación de un barrio y la apropiación de los máximos niveles de renta de suelo y sus ventajas de localización. La teoría de la gentrificación proporciona una lente relacional útil donde las injusticias asociadas a la transformación urbana capitalista pueden verse en su forma más nítida.

26 Harvey, 2007.

\section{Gentrificación, un concepto políticamente útil y "maleable"}

A pesar de sus debilidades y complejidades, sostengo que la gentrificación es una categoría conceptual útil que nos permite ser sensibles a la construcción cotidiana de la desigualdad y polarización social en la ciudad, desde dentro hacia afuera de ella. Esto implica, sin embargo, como argumentan Lees, Shin y López-Morales ${ }^{27}$ en trabajos comparativos recientes ${ }^{28}$, hay que rechazar la lógica "difusionista" que comprende la gentrificación como una cierta política neoliberal implementado en muchas ciudades, o como una ideología occidental que meramente "viaja" hacia el sur. López ${ }^{29}$ plantea se debe rechazar el tomar tan fácilmente por variables independientes o dependientes, ciertas "particularidades" alguna vez asociadas con la gentrificación en la parte occidental del hemisferio norte, a saber la supuesta agencia del consumidor de clase media-alta, el carácter post-industrial de la gentrificación, la fetichización de la escala de barrio (como si actualmente no existiesen ciudades que se gentrifican enteramente), o incuso la idea de que la gentrificación se desarrolla a través de

\footnotetext{
27 Lees, Shin y López Morales, 2015, 2016.

28 Ver López Morales, Arriagada, Gasic y Meza, 2015; Shin, Lees y López Morales, 2016; López Morales, Shin, y Lees, 2016.

29 López Morales, 2016b.
} 
ciertas "olas" o que sigue una cierta trayectoria lineal $^{30}$, una idea estructural interesante pero que a mi juicio contaba (y aún cuenta) con poca evidencia empírica.

El análisis de la disputa por la apropiación del valor de uso de los barrios (en forma de renta de suelo) también ayuda a medir y cuantificar el desplazamiento asociado a la gentrificación ${ }^{31}$. Una forma es observar, por una parte, la captura privada de la renta de suelo; por la otra, el despojo social de esta renta; lo segundo puede ocurrir a montos tan altos que no permiten a los residentes originales la permanencia o encontrar residencia de reemplazo de igual centralidad, posterior al proceso de redesarrollo de los barrios ${ }^{32}$. El enfoque de la renta de suelo da a conocer los conflictos asociados no sólo con las curvas de valor del suelo urbano, como dice Ghertner, sino también con el valor de uso de ese suelo y las posibilidades que un emplazamiento central o bien localizado ofrece a sus ocupantes, en términos de acceso a bienes públicos como áreas verde, atención de salud y educación.

Investigar la brecha de renta es una praxis que los activistas anti-gentrificación o anti-desplazamiento

\footnotetext{
30 Hackworth y Smith, 2001; Atkinson y Bridge, 2005.

31 El estudio sobre la disputa por la renta de suelo fue iniciado por Smith (1979) y Clark (1987). Luego Shin (2009a, 2009b) y López Morales $(2013,2016 \mathrm{~b})$ lo aplican para sensibilizar análisis de desplazamiento.

32

López Morales, 2013.
}

aprenden y aplican en su lucha. Hace ya bastante tiempo, la teoría de la brecha de renta y el propio Neil Smith fueron representados desde la academia como "excesivamente deterministas"33, pero esta crítica nunca entendió el verdadero poder de esta teoría que ha tenido que hacer frente a las desigualdades esencialmente neoliberales entre las fuerzas económicas y agentes sociales en muchas ciudades transformando rápidamente. Hoy las comunidades urbanas comienzan a entender el valor del conocimiento de la economía urbana, precios y márgenes de ganancias privadas (en menoscabo de los derechos sociales) que ocurren en sus barrios; algo muy patente hoy, por ejemplo, entre los activistas barriales en Ciudad de México.

Sergio González, un activista fundamental en la resistencia vecinal de la colonia Benito Juárez contra la especulación inmobiliaria en Ciudad de México, llama a la gentrificación un proceso genérico de "blanqueamiento"; esto, con el fin de incorporar tres fenómenos vinculados: la llegada de las clases acomodadas étnicamente blancas que desplazan físicamente a las comunidades de menores ingresos usualmente mestizas e indígenas, el blanqueamiento real de edificios impuestas por el gusto

33 Hamnett, 1991 
estético de la nueva clase media, y el blanqueo de dinero que permite la inversión de bienes raíces. Se trata de una definición polisémica atractiva, aún poco estudiada de México, pero que ayuda a explicar influjos importantes de inversión también en el redesarrollo urbano de toda América Latina y Estados Unidos. González comprende la utilidad política dialéctica de la teoría de la gentrificación (aunque deliberadamente no la llame como tal) porque le permite explicar los numerosos conflictos en la zona en la que vive, y no se asusta de la utilización "híbrida" del concepto al tratar de comprender (y desde ahí intervenir en) la transformación de su colonia y ciudad. Así como él, son numerosos los activistas urbanos mexicanos que comprenden, discuten difunden, distorsionan, adaptan y hacen mutar el concepto de gentrificación con el fin de aplicarlo en sus barrios.

En México, ciudad de 22 millones de habitantes, los primeros estudios de gentrificación fueron exploratorios y descriptivos, y prácticamente no tomaron en cuenta los movimientos urbanos. Desde entonces se le ha otorgado un alto valor a las políticas y planes de protección patrimonial y la inversión en actividades culturales como causas explicativas de la apropiación simbólica de las áreas centrales históricas por las clases medio-altas. Más recientemente, teóricos mexicanos han explicado una alta incidencia de políticas de remodelación turística, comercial y financiera de espacios con grados altos de centralidad, y las ventajas ofrecidas por el Estado al mercado privado de vivienda para sectores medios (Bando 2) que concentró e intensificó la re-urbanización privada en cuatro grandes áreas del centro metropolitano, además de otras directrices públicas agresivamente eficaces para desalojar la "informalidad" de espacios públicos históricos ${ }^{34}$. Sin embargo, actualmente son numerosos los nuevos casos que afloran en Ciudad de México con todo tipo de disputas de clase por el espacio, especialmente en barrios centrales. En estudio de campo realizado entre 2015 y 2016 pude constatar más de 60 en toda la Ciudad de México, casos que refieren en algún grado a aspectos asociables a gentrificación sumados a conflictos por la cada vez más escasa dotación de agua (apropiada por los grandes conjuntos que se construyen), impactos físicos y ambientales en la desvalorización de barrios, Zonas de Desarrollo Económico y Social (ZODES) que redefinen (sin participación ciudadana) completamente las normas urbanísticas en determinados lugares, megaproyectos, etc. Pero puede que, dado el dinamismo de la inversión inmobiliaria y la tremenda gravitancia que tienen

34 Jones y Varley, 1994; Hiernaux y Lindon, 2004; Delgadillo, 2014 y en prensa; Coulomb, 2012; Olivera, 2015; Janoschka, Sequera y Salinas, 2014, entre otros. 
las políticas orientadas al desarrollo urbano en la extensa parte central metrópolis, el número de casos esté desactualizado ya.

Los casos más notorios en la Ciudad de México parecieran ser el creciente interés rentista inmobiliario en colonias centrales, tales como Benito Juárez, Cuauthemoc, Doctores, Santa María La Rivera, e incluso la popular colonia Guerrero, en un perímetro muy cercano al centro, y el sector Granadas, hoy rebautizado como "Nueva Polanco" por los desarrolladores y en los planes urbanos del gobierno de la Ciudad de México ${ }^{35}$. Allí, se dan situaciones tan específicas como el alto número de propiedades "intestadas" (propiedades parcial o totalmente sin herederos legalmente registrados) y la ocupación consuetudinaria que durante décadas han ejercido familias de inquilinos, que fueran en la década de 1940 beneficiados por leyes progresistas de control de rentas. Al haber sido eliminadas estas leyes durante el sexenio del gobierno de Salinas de Gortari (1988-1994), un número creciente de inmobiliarias descubrieron que podían, mediante la aplicación de arreglos legales no siempre transparentes, incentivar a propietarios ausentes a cederles derechos de propiedad con una promesa de compra y hacerse cargo de "incrementar" las rentas y/o incentivar a inquilinos a que desalojen. En paralelo, las inmobiliarias compran locales comerciales los barrios de moda, y de esta forma también "reajustan" la oferta de atractivos culturales consumibles para la nueva población, tales como bicicletas, cafetería y gastronomía gourmet, ropa, accesorios, galerías de arte, etc. Hoy los precios de oferta de un departamento nuevo de $50 \mathrm{~m}^{2}$ llegan fácilmente a 4 millones de pesos mexicanos, alrededor de US\$220.000 dólares, ó \$150 millones de pesos chilenos. Una oferta inalcanzable para la gran mayoría de inquilinos y ocupantes originales de esas colonias (figura 1).

Y luego están las macro-operaciones urbanas dirigidas por conglomerados público-privados, tales como la recientemente creada Corporación ProCDMX, legado del actual Jefe de Gobierno del ex Distrito Federal, Miguel Ángel Mancera, y su principal asesor en materias de desarrollo urbano, Simón Levy, quienes intentaron imponer el nuevo "Corredor Cultural Chapultepec" en pleno centro metropolitano. Esta iba a ser una de las obras urbanas de mayor impacto en la ciudad, un corredor de transporte y servicios en el tramo entre Bosque de Chapultepec y Glorieta Insurgentes, que en términos simples contemplaba el hundimiento de la calzada, la edificación de una vía motorizada concesionada (de pago) en segundo piso, y un mega shopping mall de 5 plantas en el centro de la vía. Tras una intensa campaña ciudadana llevada principalmente adelante por organizaciones vecinales de esas zonas, se realizó un plebiscito ciudadano

Delgadillo, 2014; Salinas, 2013. 
FIGURA 1. LOFTS, BICICLETAS Y TIENDAS GOURMET EN LA COLONIA BENITO JUÁREZ, CIUDAD DE MÉXICO.

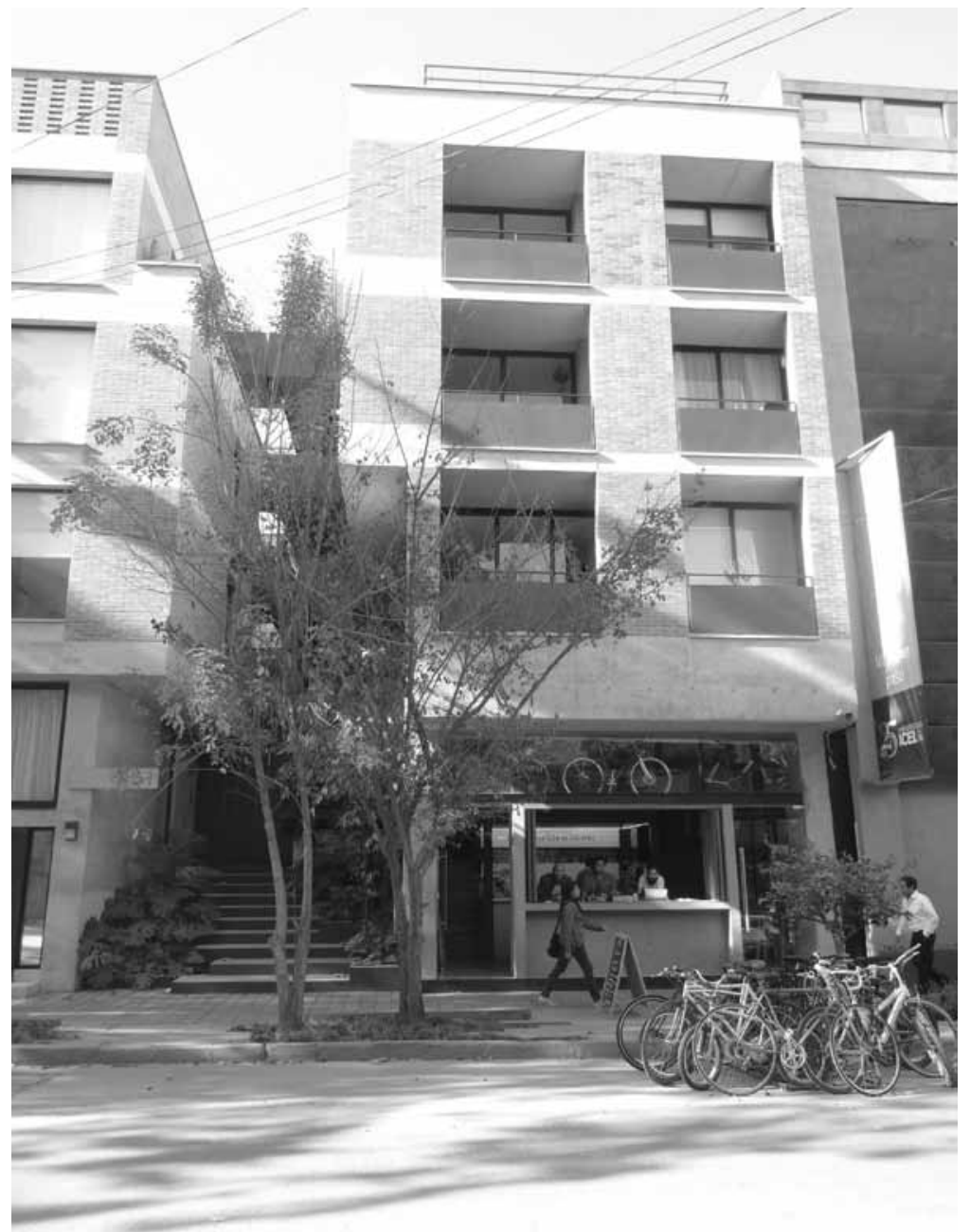

Fuente: Autor. 
el 6 de diciembre de 2015, que rechazó con más de un $65 \%$ de los votos este proyecto, rechazo mayúsculo ante un modelo de gestión urbana que durante los dos años previos no había considerado más que canales débilmente informativos hacia la población, y una propuesta de desarrollo urbano que entregaba por un plazo de 30 años a tres conglomerados inmobiliarios-bancarios el monopolio de los bordes de la vía y del shopping mall. El plebiscito del 6 de diciembre de 2015 no sólo reflejó el creciente poder de organización y resistencia vecinal que se ha desarrollado en la ciudad, sino que es uno más de las formas en que se refleja la pérdida generalizada de confianza en las instituciones públicas y del Estado en ese país.

México demuestra como la informalidad en la ocupación y tenencia de vivienda y suelo sitúa al residente en una posición sólo parcialmente más desventajada respecto al residente "formal". Por una parte, mientras la informalidad en la tenencia de la vivienda es un importante acelerador para la especulación con propiedades, el desalojo y desplazamiento es naturalizado como un resultado inevitable de la modernización, es decir, existe una relación entre la tenencia informal del suelo y propiedades y la aceleración de los procesos especulativos promovido tanto desde el exterior (empresarios privados y el Estado) y el interior (empresarios locales) de los enclaves sociales en proceso de recambio urbano ${ }^{36}$. Por la otra, las redes vecinales de resistencia y construcción de alternativas a la especulación rentista se configuran por una amplia variedad de sujetos residentes que responden a situaciones diversas de tenencia y ocupación, ya que comprenden que su situación depende fundamentalmente de los contradictorios arreglos legales (ley de control de rentas versus neoliberalización del mercado de propiedades), impuestos desde el Estado en ya casi el último siglo. Existe por lejos una mayor organización vecinal en Ciudad de México que en la mayoría de los lugares gentrificados de América Latina, con formas no sólo aun relativamente independientes de las agendas políticas de partidos establecidos (principalmente son los partidos derechistas PAN y PRI, y los más recientes herederos de "izquierda" de la estructura institucional del PRI denominados PRD y MORENA) sino que también despliegan modalidades de organización y activismo en red muy eficientes y con un alto manejo mediático (figura 2). También manejan como elemento de discurso fundamental la preservación patrimonial arquitectónica y de diseño urbano de importantes barrios de la ciudad, pero no son "patrimonialistas", es decir no están disociados de otras consideraciones tales como la defensa de los sujetos sociales que habitan en estos espacios, tal como ocurre en Chile.

$36 \quad$ Ley y Teo, 2014. 
FIGURA 2. AFICHE DE CAMPAÑA SOCIAL CONTRA EL CORREDOR CULTURAL CHAPULTEPEC, DISEÑADO POR ACTIVISTAS DE LA COLONIA JUÁREZ (PLATAFORMA VECINAL 066600) Y DE AMPLIA DIFUSIÓN EN LA CIUDAD.

\section{PROTEJAMOS NUESTRA CIUDAD EL 6 DE DICIEMBRE VOTA}

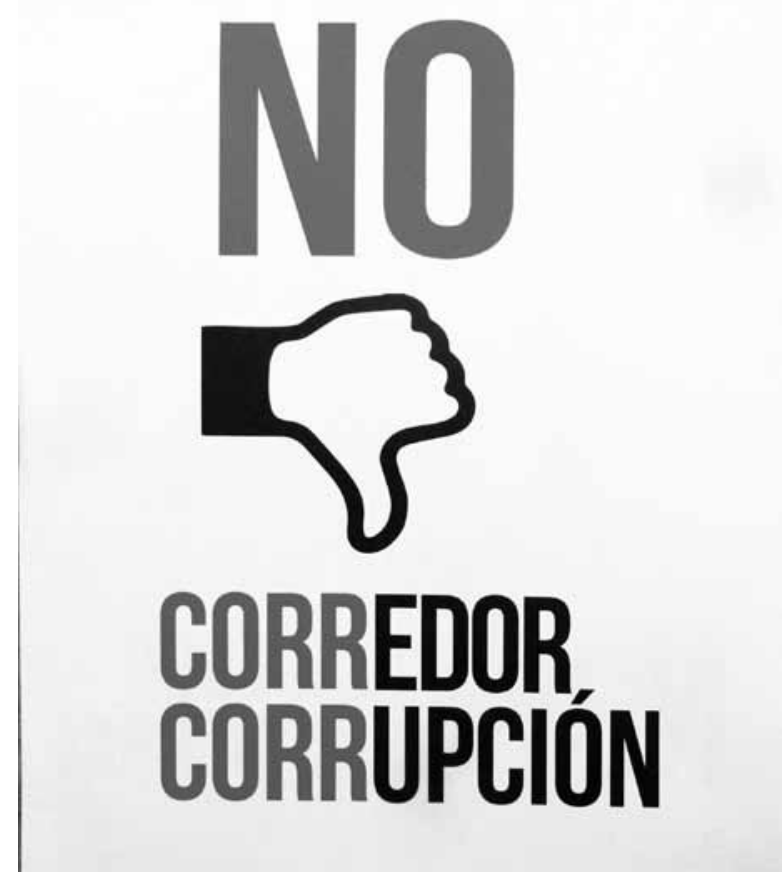

Fuente: Autor
En el caso de Chile, el término de "gentrificación" no se utiliza por los movimientos barriales patrimonialistas, pero sí es políticamente utilizado por el Movimiento de Pobladores en Lucha (MPL) ${ }^{37}$ y el Ukamau ${ }^{38}$ para estructurar sus reivindicaciones no sólo de vivienda social de calidad sino de suelo central para permanecer y desplegar estrategias de consolidación de barrios populares en suelo central, sin que por ellos deban ser sujetos partícipes de un mercado de especulación rentista. Como alguna vez definiera Amparo García, líder comunitaria portorriqueña, "no es que nos guste vivir así, es que queremos vivir aquí". Es decir, tampoco es legítimo que se fuerce a los pobres urbanos a optar al hacinamiento y la peligrosidad de habitar viviendas envejecidas como moneda de cambio por su permanencia en áreas centrales. Es vital se entienda el derecho al hábitat o a la vivienda digna es también un derecho a la centralidad. La discusión sobre gentrificación en Chile apunta especialmente hacia este último punto.

Las comunas centrales de Santiago, el área metropolitana más grande de Chile de casi 7 millones de habitantes, son un ejemplo interesante de cómo la gentrificación está estrechamente correlacionada con las formas en que el capitalismo contemporáneo rehace la ciudad, y el Estado activamente aumenta la posibilidad de acumular capital a través de la explotación del suelo para construcción y

\footnotetext{
37 Pérez, 2016.

38 http://mp-ukamau.blogspot.cl
} 
la recomposición socio-económica de los barrios, atrayendo bolsas de consumidores de vivienda y desplazando a los residentes originales que quedan "fuera del mercado"39. La gentrificación del espacio central probablemente es el dispositivo más rápido para amplificar el capital invertido en el entorno construido. El espacio central es a la vez un producto y un mecanismo de captura de la plusvalía de renta de suelo, proceso expansivo que responde a las fluctuaciones económicas con períodos de inversión y acumulación, ya sea antecediendo o con posterioridad a crisis financieras globales y locales. Por ejemplo, el volumen de inversión y producción inmobiliaria en espacios centrales de Santiago aumentó dramáticamente después de la crisis asiática de 1998; luego, algo similar ocurrió tras la crisis de Wall Street en $2008^{40}$.

La naturaleza de la renta de suelo que se explota a través de este tipo de procesos reconstitutivos de la ciudad, y el tremendo poder que tiene la brecha de renta de suelo para atraer capital y esfuerzos del sector público articulados con el mercado, desde sólo hace poco tiempo que se discute en detalle suficiente en los círculos académicos globales, pero es algo que ha estado pasando en Chile desde al menos la década de 1990. Si Neil Smith y Eric Clark vieron en su momento la devaluación concertada de propiedades como una forma de

39 López Morales, 2013, 2015, 2016 b.

40 López Morales, 2016. ampliar la brecha de renta, en Chile el Estado y las inmobiliarias han "estirado" la brecha de renta desde el otro extremo, es decir, aumentando la renta potencial obtenible por los agentes desarrolladores privados a través la amplificación de los Coeficientes de Constructibilidad (Coeficiente de Utilización del Suelo CUS en México, Coeficiente de Ocupación Total en Argentina, Floor Area Ratio en países anglosajones, etc.), que es la capacidad de construir una superficie total derivado del tamaño de la tierra sobre la cual se construye el edificio ${ }^{41}$.

En teoría quienes sean propietarios originales deberían tener la posibilidad de vender sus predios a las inmobiliarias a precio de mercado, y utilizar ese dinero para encontrar residencia de reemplazo en similares o mejores condiciones. En teoría también, los hogares arrendatarios que ocupan vivienda antigua deberían tener posibilidades de acceder a arrendar en viviendas nuevas construidas. Sin embargo, en la práctica, ocurren tres cosas muy distintas. Primero, cuando propietarios-residentes originales venden sus propiedades de suelo a las inmobiliarias, a menudo sucede que el precio no deriva de una negociación sino de una imposición de precio o monopsonio (donde varios vendedores se enfrentan a un solo comprador, es decir, una inmobiliaria) que reduce el precio efectivo de su parcela, y por ende descapitaliza a ese propietario y limita 
sus opciones de ocupación posterior ${ }^{42}$. Segundo, el encarecimiento sostenido en más de 100\% de los precios de venta y arriendo de vivienda nueva en las comunas centrales y en general en todo Santiago, que se han observado desde el año 2000 de más de $100 \%$, hacen que exista un incremento similar también en los precios de arriendo, por lo que abandonar una propiedad central significa perder un activo de buena localización a precio razonable; acceso al empleo, servicios y bienes públicos; y redes sociales barriales establecidas, y ser desplazado a comunas con menor valorización debido a su menor centralidad y menor oferta de bienes públicos, parques, educación y acceso a centros de salud. Y tercero, existe una pérdida de espacio útil doméstico y de uso de patio, para fines productivos y recreativos, y la posibilidad de residir integrado a redes vecinales como ocurre en los barrios residentes de origen, y no en condominios verticales donde la vida barrial desaparece.

La estructura de poder conjunto del sector inmobiliario impone estas condiciones en las comunas interiores de la ciudad; es decir, el sistema depende de la dominación de los conglomerados (usualmente, las empresas inmobiliarias más grandes del país y que cotizan en la bolsa) que poseen las más altas capacidades tecnológicas y financieras orientadas a la construcción. Sólo unas pocas empresas dominan el mercado (al año 2012, en el explotado barrio de

42 López Morales, Arriagada, Gasic y Meza, 2015.
Santa Isabel, en la comuna central de Santiago, el 50 por ciento de la oferta era producida por cuatro empresas inmobiliarias de gran escala $)^{43}$.

Las pérdidas patrimoniales urbanas son considerables, especialmente en lo referido a la pérdida de imagen de los barrios. Esto lo conocen bien, y lo han situado en su agenda principal, el creciente número de movimientos de defensa patrimonial (sólo por citar dos, Yungay y Matta Sur). Pero la mercantilización y privatización del suelo expulsa una parte importante de los habitantes originales modestos del centro de Santiago, como efecto principal. Toda una reestructuración sociológica está llevándose a cabo en Santiago, pero los que dominan este sistema de producción inmobiliaria de la gentrificación no son una cierta fracción de la clase media, como incorrectamente arguyeron en su momento Maloutas y Hamnett, sino que son los empresarios inmobiliarios e indirectamente sus inversionistas, potentes especuladores a gran escala con suficiente poder para incidir en las normativas urbanas, comprar, acumular, re-vender, desarrollar el suelo y producir vivienda a precios controladamente caros, porque también tienen capacidad de dominar el volumen global de la producción de oferta. La visualidad del marketing inmobiliario también denota el perfil "blanqueado" de los usuarios de estos nuevos departamentos, al decir de Sergio González. El arribo de población soltera

43 López Morales, 2013. 
FIGURA 3. IMAGEN “BLANQUEADA” EN SAN MIGUEL, UNA COMUNA CENTRAL TRADICIONALMENTE POPULAR DE SANTIAGO.

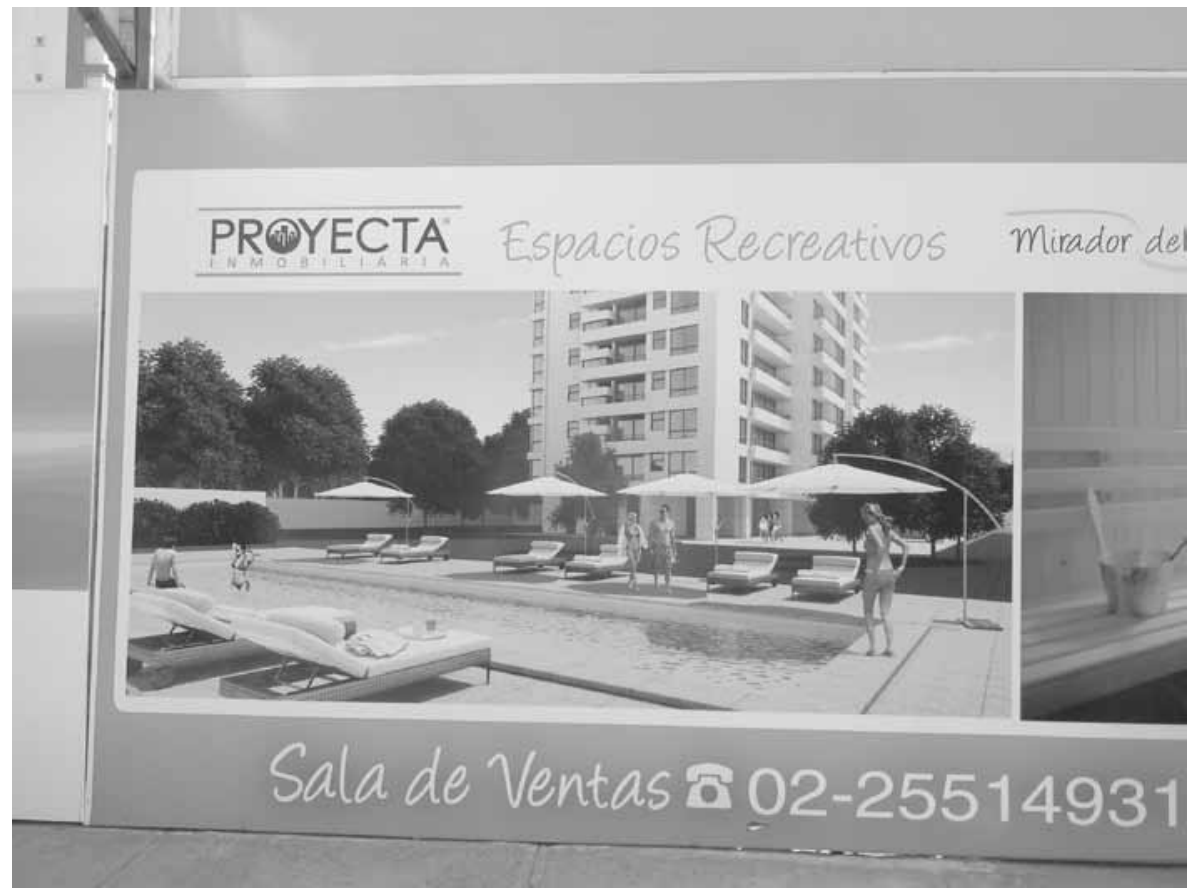

Fuente: Autor

o sin hijos, con estudios universitarios e ingresos de hogar más altos, ha sido incremental (figura 3).

Los potentes sectores inmobiliario y financiero abogan frente al Estado por una serie de prescripciones de políticas pro-empresariales. El subsidio de "Renovación Urbana", que en términos simples ascendía al $10 \%$ del precio de vivienda, aplicado mayormente en comunas centrales, provino de una alianza municipal-privada que en 1990 demandó al Estado esta medida. En pocos años, este 
subsidio se aplicaba a la compra de más del 95\% de la vivienda ofrecida. Actualmente, la cobertura del subsidio es inferior al 1\%. Sin embargo, al año 2015 las inmobiliarias chilenas produjeron un sobre-stock de vivienda a niveles nunca vistos en la historia del país, un sobre calentamiento del mercado residencial generado por la última reforma tributaria en el país que aceleró el proceso de construcción y compra con ventajas fiscales existente hasta el año 2015, proceso que por su volumen no tuvo precedentes en el país ${ }^{44}$. El sobre stock ha sido amasado y hoy supera largamente la real demanda de vivienda existente en Chile. Ergo, durante varios años posteriores, el subsidio al arriendo ya existente desde 2013 posibilitará que bancos, inversionistas e inmobiliarias no se descapitalicen ya que la demanda real por vivienda se desplazará al nuevo nicho subsidiado: el mercado del arriendo ${ }^{45}$. Lo anterior es una semblanza muy escueta de dos políticas pro-empresariales chilenas, que posibilitan la máxima acumulación de brecha de renta.

\section{Conclusión}

Pese a la evidencia anterior, y contrariamente a lo que argumentan críticos actuales, la gentrificación está lejos de ser solamente el resultado de fuerzas económicas sobre los barrios. La gentrificación es

44 Asociación de Bancos e Instituciones Financieras, 2015.

45 López Morales, 2016. un conflicto político. Es uno de los procesos más importantes y socialmente injustos que afectan a un número creciente de ciudades hoy en día. La teoría de la gentrificación es útil porque nos impulsa a discutir y confrontar la evidencia de los procesos de rampante mercantilización y explotación de la ciudad en todas partes. Es útil también porque implica revisar siempre la tensión de clase y la polarización de poder existentes detrás de cada proceso de inversión económica y transformación urbana. Requiere incluso de una forma relacional de analizar el desarrollo urbano, que no se comprenden solamente desde una perspectiva positivista que describe agentes con poder de consumo diferenciado, sino desde una perspectiva dialéctica que comprende cómo el accionar de agentes con desigual capital social, económico y cultural están interconectados en sus esfuerzos por ocupar un determinado lugar en el espacio de la ciudad. La gentrificación no se trata sólo de brechas de renta de suelo incrementadas, sino que se trata de la pérdida de valor de uso en el espacio, así como la denegación del derecho de uso de determinados espacios para la reproducción social de las capas sociales más bajas, en cuanto el valor de cambio de esa tierra aumenta hasta el punto de que cualquier otro valor consideración se vuelve irrelevante para aquellos que toman decisiones unilaterales sobre el desarrollo de la ciudad. 
Incluso más allá de la explotación de suelo, si la teoría de la gentrificación tiene algo importante que contribuir a los estudios urbanos, es precisamente su perspectiva relacional de clase sobre el recambio urbano; es decir, la capacidad que entrega al investigador urbanista para ver simultáneamente las partes en conflicto, las estrategias y tácticas desplegadas, en procesos donde el recambio urbano está monopolizado por ciertos objetivos motivado de rentabilización privada, con la activa participación del Estado como agente principal generador de condiciones favorables al recambio de clase, y con ello la desaparición de elementos constitutivos de barrio importantes para un hábitat popular. La gentrificación es entonces un problema político, y, obviamente, no un estructuralismo económico o una idea meramente centrada en la propiedad del suelo.

Siendo así, debemos evitar el demasiado particularista supuesto post-colonial de que la teoría urbana del así llamado "norte global" debe ser automáticamente descartada, por su escasa utilidad hermenéutica, en las realidades del sur global. Si tal fuera el caso, no tendríamos teorización en las ciencias sociales en absoluto. Esta es una posición que no ayuda a la teoría social urbana a evolucionar, y tiene el efecto contrario al deseado, confinando la academia a una teorización particularizada a cada zona del mundo. Los investigadores, activistas, pensadores, ciudadanos del así llamado "sur global" necesitamos la plena libertad de nuestra imaginación, incluso si esto incluye la búsqueda de utilidad en "viejas" ideas provenientes del "norte", independientes de las olas de cierta culpa postcolonial que caracteriza ciertos investigadores del hemisferio norte, y de las naturales evoluciones epistemológicas que se experimenten en las academias de los países industrializados.

Como lo muestra el caso de Santiago, la teoría de la brecha de renta (que se aplicó por primera vez en Europa y los EEUU en la década de 1980) hoy ayuda a explicar muchas otras situaciones diferentes. Esto incluye a un importante número de propietarios-residentes, arrendatarios y ocupantes formales o informales, minorías que también son agentes urbanos y tienen derecho a la inclusión en las políticas de vivienda y ciudad, pero que por el contrario son excluidos de las definiciones normativas que impone el mercado en la reconfiguración de sus "nichos" de mercado. Ciudad de México también aporta una observación importante. El Estado requiere ser mejor estudiado y comprendido como un agente agresivo y decisivo en la creación de la gentrificación; esto aplica no sólo a México u otros países del así llamado sur global, sino también incluso a los países industrializados del norte, como visualizan en un alto número de casos en el trabajo comparativo Lees, Shin y López-Morales ${ }^{46}$ (2015,

46 Lees, Shin y López Morales, 2015, 2016. 
2016). Parte importante de la movilidad transnacional del capital, transferencia de políticas públicas, y las posibilidades de compartir teoría urbana a niveles y escalas sin precedentes, constituye el carácter "planetario" que asume actualmente el concepto de la gentrificación.

Ergo, si nos hemos de quedar atrapados en definiciones restrictivas, meramente descriptivas o poco críticas de la gentrificación, ni los casos de Chile, México, la India o China, ni la actual gentrificación dirigida por el Estado en Londres y otras ciudades de Inglaterra, encajarían en esa definición. Sin embargo, una teorización suficientemente sensible como para reconocer que la gentrificación también significa desigualdades urbanas y procesos de segregación acentuados por el Estado en respuesta a los intereses privados a gran escala, tanto transnacionales como locales o mutuamente imbricados, puede proporcionar herramientas intelectuales y políticas útiles para la mayor comprensión de las transformaciones físicas y socioeconómicas de los entornos urbanos, que ocurren diversamente en el mundo actual. La gentrificación es planetaria, útil, adaptable y políticamente relevante.

\section{Bibliografía}

ASOCIACIÓN de Bancos e Instituciones Financieras.

Colocaciones hipotecarias: crecimiento estable e indicadores de riesgo acotados. [En línea].
ABIF. 2015. [Fecha de consulta: 18 febrero 2016]. Disponible en: http://www.abif.cl/wp-content/ uploads/2015/09/INFORME-ABIF-N-59-Colocaciones_hipotecarias.pdf

ATKINSON, Rowland, ed. y BRIDGE, Gary, ed. Gentrification in a global context: The new urban colonialism. Londres, Routledge. 2005.

BETANCUR, John. Gentrification in Latin America: overview and critical analysis. Urban Studies Research. 2014. DOI 10.1155/2014/986961.

CLARK, Eric. The Rent Gap and Urban Change: Case Studies in Malmö 1860-1985. Lund, Lund University Press. 1987.

COULOMB, René. El centro de la ciudad de México frente al desafío de un desarrollo urbano más sustentable. En COULOMB, René, ed.; ESQUIVEL, María Teresa, ed. y PONCE, Gabriela, ed. Hábitat y centralidad en México: un desafío sustentable Mexico, Centro de Estudios Sociales y de Opinión Pública. 2012. p. 9-16. ISBN 978-607-7919-30-8.

CUMMING, Jake. Confronting Favela Chic: the Gentrification of Informal Settlements in Rio de Janeiro, Brazil. En: LEES, Loretta, ed.; SHIN, Hyun Bang, ed. y LÓPEZ MORALES, Ernesto, ed. Global Gentrifications: Uneven Development and Displacement. Bristol, Policy Press. 2015. p. 81-99.

DELGADILLO, Víctor. Ciudad de México: megaproyectos urbanos, negocios privados y resistencia social. En: HIDALGO, Rodrigo, ed. y JANOSCHKA, Michael, ed. La ciudad neoliberal: gentrificación y exclusión en Santiago de Chile, Buenos Aires, 
Ciudad de México y Madrid. Santiago, Universidad Católica de Chile. 2014. p. 199-215.

DELGADILLO, Víctor. Selective modernization of Mexico City and its Historic Center. Gentrification without Displacement? Urban Geography. DOI 10.1080/02723638.2015.1096114. En prensa.

DOSHI, Sapana. The politics of the evicted: Redevelopment, subjectivity, and difference in Mumbai's slum frontier. Antipode. 45(4): 844-865, 2013. DOI 10.1111/j.1467-8330.2012.01023.x.

GAYTÁN, Pablo y PIMENTEL, Fabián. Privatización del espacio público en la ciudad de México: de la ciudad moderna a la segregación socioespacial neoliberal. México, Colegio de antropología Social de la Benemérita Universidad Autónoma de Puebla. En prensa.

GAFFNEY, Chris. Gentrifications in pre-Olympic Rio de Janeiro. Urban Geography. 2015. DOI 10.1080/02723638.2015.1096115.

GHERTNER, Asher. Why gentrification theory fails in 'much of the world'. City. 19(4): 552-563, 2015. DOI 10.1080/13604813.2015.1051745.

HACKWORTH, Jason y SMITH, Neil. The changing state of gentrification. Tijdschrift poor Economische en Sociale Geografie. 92(4): 464-477, 2001. DOI 10.1111/1467-9663.00172.

HAMNETT, Chris. The Blind Men and the Elephant: The Explanation of Gentrification. Transactions of the Institute of British Geographers. 16(2): 173-189, 1991. DOI 10.2307/622612.
HARVEY, David. A brief history of neoliberalism. Oxford, Oxford University Press. 2007.

HIERNAUX, Daniel y LINDÓN, Alicia. Desterritorialización y reterritorialización metropolitana: la ciudad de México. Documents d'Anàlisi Geogràfica. (44): 71-88, 2004.

JANOSCHKA, Michael, SEQUERA, Jorge y SALINAS, Luis. Gentrification in Spain and Latin Ameri$\mathrm{ca}-\mathrm{a}$ Critical Dialogue. International Journal of Urban and Regional Research. 34(4): 1234-1265, 2014. DOI 10.1111/1468-2427.12030.

JONES, Gareth y VARLEY, Ann. The Contest for the City Centre: Street Traders versus Buildings. Bulletin of Latin American Research. 13(1): 27-44, 1994. DOI $10.2307 / 3338700$.

LEES, Loretta. The urban injustices of New Labour's 'new urban renewal': the case of the Aylesbury Estate in London. Antipode. 46(4): 921-947, 2014. DOI 10.1111/anti.12020.

LEES, Loretta, ed.; SHIN, Hyun Bang, ed. y LÓPEZMORALES, Ernesto, ed. Global Gentrifications: Uneven Development and Displacement. Bristol, Policy Press. 2015.

LEES, Loretta, SHIN, Hyun y LÓPEZ-MORALES, Ernesto. Planetary Gentrification. Cambridge, Polity Press. 2016.

LEY, David y TEO, Sin Yih. Gentrification in Hong Kong? Epistemology vs. ontology. International Journal of Urban and Regional Research. 38(4): 1286-1303, 2014. DOI 10.1111/1468-2427.12109. 
LÓPEZ MORALES, Ernesto. Gentrification in Chile: A property-led process of dispossession and exclusion in the inner city of Santiago. Urban Geography. 2016a. DOI 10.1080/02723638.2016.1149311.

--- Assessing exclusionary displacement through rent gap analysis in the urban redevelopment of inner Santiago, Chile. Housing Studies. 31(5): 540-559, 2016b. DOI 10.1080/02673037.2015.1100281.

--- Gentrification in the global South. City. 19(4): 557566, 2015. DOI 10.1080/13604813.2015.1051746.

--- Gentrificación en Chile: aportes conceptuales y evidencia para una discusión necesaria. Revista de Geografía Norte Grande. (56): 31-52, 2013. DOI $10.4067 / 50718-34022013000300003$.

--- Gentrification by Ground Rent Dispossession: The Shadows Cast by Large-Scale Urban Renewal in Santiago de Chile. International Journal of Urban and Regional Research. 35(2): 330-357, 2011. DOI 10.1111/j.1468-2427.2010.00961.x.

--- Real Estate market, state-entrepreneurialism and urban policy in the 'gentrification by ground rent dispossession' of Santiago de Chile. Journal of Latin American Geography. 9(1): 145-173, 2010. DOI 10.1353/lag.0.0070.

LÓPEZ MORALES, Ernesto; ARRIAGADA, Camilo; GASIC, Ivo y MEZA, Daniel. Efectos de la renovación urbana sobre la calidad de vida y perspectivas de relocalización residencial de habitantes centrales y peri centrales del Área Metropolitana del Gran Santiago. EURE. 41(124): 45-67, 2015. DOI 10.4067/S0250-71612015000400003.
LÓPEZ MORALES, Ernesto; GASIC, Ivo y MEZA Daniel. Urbanismo pro-empresarial en Chile: políticas y planificación de la producción residencial en altura en el pericentro del gran Santiago. Revista INVI. 28(76): 75-114, 2012. DOI 10.4067/ S0718-83582012000300003.

LÓPEZ MORALES, Ernesto; SHIN, Hyun Bang y LEES, Loretta. Latin American gentrifications. Urban Geography. 2016. DOI 10.1080/02723638.2016.1200335.

MALOUTAS, Thomas. Contextual diversity in gentrification research. Critical Sociology. 38(1): 33-48, 2011. DOI 10.1177/0896920510380950.

MCFARLANE, Colin. The comparative city: knowledge, learning, urbanism. International Journal of Urban and Regional Research. 34(4): 725-742, 2010. DOI 10.1111/j.1468-2427.2010.00917.x.

MCFARLANE, Colin y ROBINSON, Jennifer. Introduction-Experiments in Comparative Urbanism. Urban Geography. 33(6): 765-773, 2012. DOI 10.2747/0272-3638.33.6.765

MUELLER, Gavin. Liberalism and Gentrification. [En línea]. Jacobin. 26 septiembre 2014. [Fecha de consulta: 18 febrero 2016]. Disponible en https://www.jacobinmag.com/2014/09/ liberalism-and-gentrification.

OLIVERA, Patricia. Gentrificación en la ciudad de México, entre políticas públicas y agentes privados. En: DELGADILLO Victor, ed.; DÍAZ, Ibán, ed. y SALINAS, Luis, ed. Perspectivas del estudio de la 
gentrificación en México y América Latina. Ciudad de México, UNAM. 2015. p. 91-110.

PÉREZ, Miguel. A new poblador is being born: Housing struggles in a gentrified space of Santiago, Chile. Latin American Perspectives. 2016. DOI 10.1177/0094582X16668318.

POSSO, Ladys. Patrimonialización, especulación inmobiliaria y turismo: gentrificación en el barrio Getsemaní. En: DELGADILLO Victor, ed.; DÍAZ, Ibán, ed. y SALINAS, Luis, ed. Perspectivas del estudio de la gentrificación en México y América Latina. Ciudad de México, UNAM. 2015. p. 175-190.

ROBINSON, Jennifer. Cities in a world of cities: the comparative gesture. International Journal of Urban and Regional Research. 35(1): 1-23, 2011. DOI 10.1111/j.1468-2427.2010.00982.x.

ROY, Ananya. The 21st-Century Metropolis: New Geographies of Theory. Regional Studies. 43(6): 819830, 2009. DOI 10.1080/00343400701809665.

SALINAS, Luis. La gentrificación de la Colonia Condesa, Ciudad de México. Aporte para una discusión desde Latinoamérica. Revista Geográfica de América Central. 2(51): 116-138, 2013. ISSN 2215-2563.

SHIN, Hyun. Contesting speculative urbanisation and strategising discontents. City. 18(4-5): 509-516, 2014. DOI 10.1080/13604813.2014.939471.

--- Property-based redevelopment and gentrification: The case of Seoul, South Korea. Geoforum. 40(5): 906-917, 2009a. DOI 10.1016/j. geoforum.2009.06.009.
--- Residential redevelopment and entrepreneurial local state: The implications of Beijing's shifting emphasis on urban redevelopment policies. Urban Studies. 46(13): 2815-2839, 2009b. DOI 10.1177/0042098009345540.

SHIN, Hyun y KIM, Soo-Hyun. The developmental state, speculative urbanisation and the politics of displacement in gentrifying Seoul. Urban Studies. 53(3): 540-559, 2016. DOI: 10.1177/0042098014565745.

SHIN, Hyun; LEES, Loretta y LÓPEZ-MORALES, Ernesto. Introduction: Locating gentrification in the Global East. Urban Studies. 53(3): 455-470, 2016. DOI 10.1177/0042098015620337.

SMITH, Neil. New globalism, new urbanism: Gentrification as global urban strategy. Antipode. 34(3): 427-450, 2002. DOI 10.1111/1467-8330.00249.

--- Toward a theory of gentrification: A back to the city movement by capital not people. Journal of the American Planning Association. 45(4): 538-548, 1979. DOI 10.1080/01944367908977002.

SPIVAK, Gayatri. Death of a Discipline. Nueva York, Columbia University Press. 2003. 


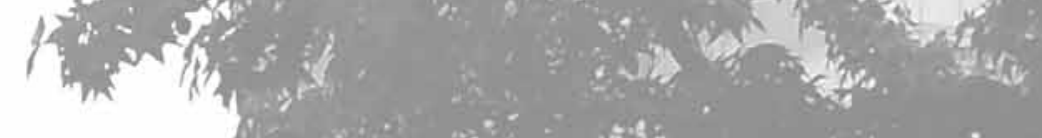

fi

\section{INFORMATIVO / INFORMATION}

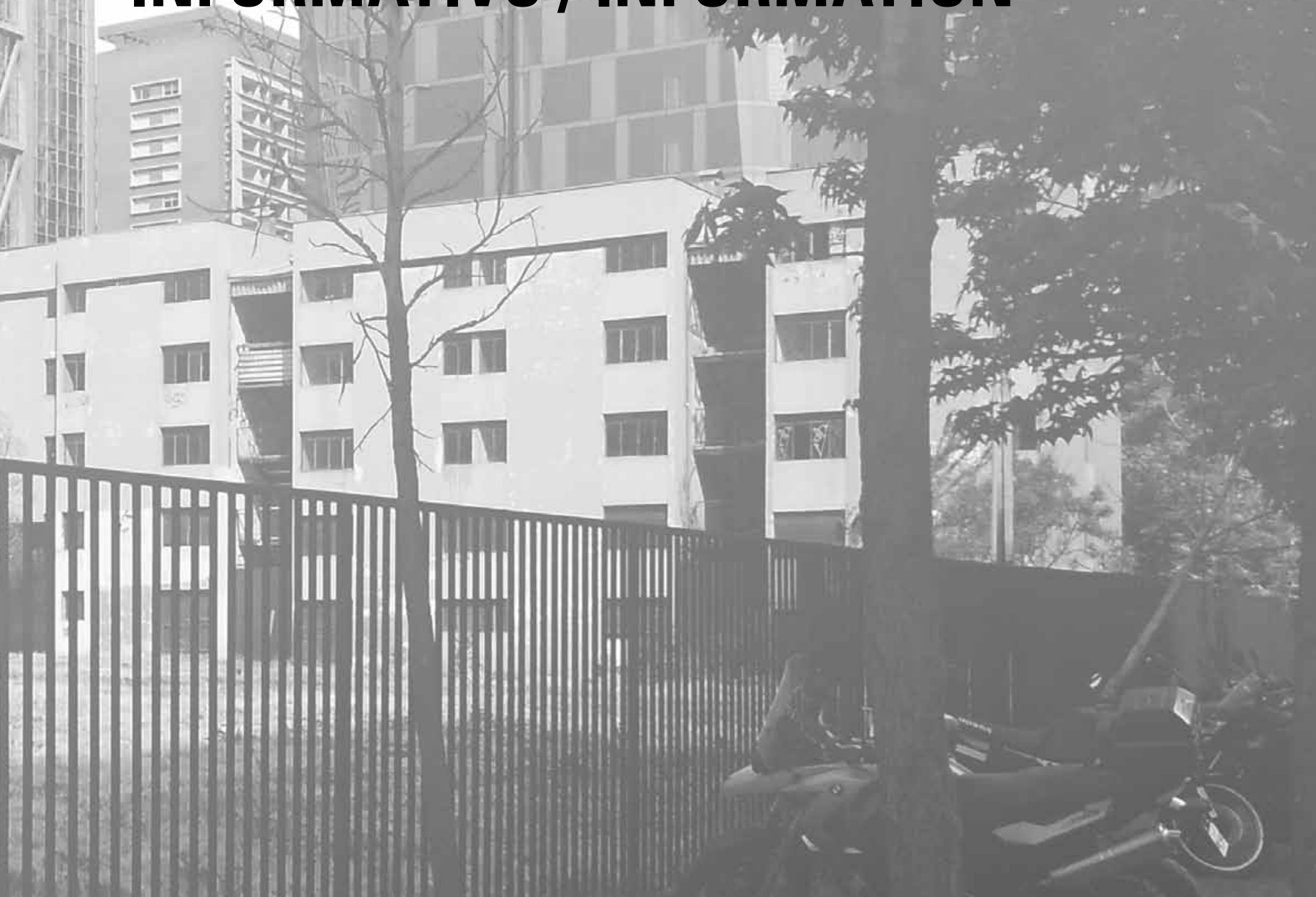

